\title{
New theories for the Fermi scale
}

\section{Christophe GROJEAN*}

CERN, Physics Department, Theory Unit, Geneva, Switzerland and Institut de Physique Théorique, CEA Saclay, France

E-mail: christophe.grojean@cern.ch

Electroweak interactions need three Nambu-Goldstone bosons to provide a mass to the $W^{ \pm}$and the $Z$ gauge bosons but they also need an ultra-violet (UV) moderator or new physics to unitarize the gauge boson scattering amplitudes. In this talk, I will present various recent models of physics at the Fermi scale: several deformations of the Minimal Supersymmetric Standard Model, Little Higgs models, holographic composite Higgs models, 5D Higgsless models.

European Physical Society Europhysics Conference on High Energy Physics July 16-22, 2009

Krakow, Poland

* Speaker. 


\section{The Standard Model and the mass problem}

The strong, weak and electromagnetic interactions of elementary particles are described by gauge interactions based on a symmetry group $S U(3)_{c} \times S U(2)_{L} \times U(1)_{Y}$. Gauge theory is not only a way to classify particles and assign quantum numbers to them but it is also a dynamical principle that predicts particular couplings among particles. And the structure of these interactions has been well tested at LEP, for instance in the process $e^{+} e^{-} \rightarrow W^{+} W^{-}$. While this is certainly true at least for the 3-point functions, namely the interactions involving at least three particles, the gauge structure is actually badly violated at the level of the 2-point functions, namely in the mass spectrum: the observed mass terms for the leptons and the gauge bosons are not gauge invariant since the gauge group is chiral and also acts non-linearly on the gauge fields. This apparent clash calls for a spontaneous breaking of the gauge symmetry.

In the broken phase, a (massive) spin one particle describes three different polarizations: two transverse ones plus an extra longitudinal one which decouples in the massless limit. In the Standard Model (SM), the longitudinal degrees of freedom associated to the $W^{ \pm}$and $Z^{0}$ gauge bosons correspond presumably to the eaten Nambu-Goldstone bosons resulting from the breaking of the global chiral symmetry $S U(2)_{L} \times S U(2)_{R} / S U(2)_{V}$. This picture still leaves us with the question of the source of the Nambu-Goldstone bosons: What is the sector responsible for the breaking $S U(2)_{L} \times S U(2)_{R} \rightarrow S U(2)_{V}$ ? What is the dynamics of this sector? What are its interactions with the SM particles? The common lore is that these extra degrees of freedom are part of a fundamental scalar field transforming as a weak doublet. This Higgs doublet corresponds to 4 real scalar fields: the 3 eaten Nambu-Goldstone bosons and one physical real scalar degree of freedom, the notorious Higgs boson. While this picture is in very good agreement with Electroweak (EW) data ${ }^{1}[1,2,3]$, the very fact that its unique prediction, namely the existence of the Higgs boson, has not been verified experimentally yet leaves open the possibility for other origins of the Nambu-Goldstone bosons: e.g., condensates of techniquarks, components of some gauge fields along an extra dimension ...

\section{The Higgs boson: a simple picture that calls for new physics}

The Higgs mechanism is at best a description, but certainly not an explanation, of electroweak symmetry breaking (EWSB) since there is no dynamics that would explain the instability of the Higgs potential at the origin. Moreover, it also jeopardizes our current understanding of the SM at the quantum level since the Higgs potential suffers from two sources of radiative instabilities: (i) the mass term is quadratically divergent while (ii) the quartic Higgs-self interactions could easily be driven to a Landau pole or to a rolling vacuum at very large value of the Higgs field if the Higgs mass does not lie in the window around 130 and $170 \mathrm{GeV}$. New physics is required to solve these "gauge hierarchy", "triviality" and "(meta)stability" problems.

There are of course additional arguments for the existence of new physics beyond the SM: (i) at the level of $2 \div 3$ standard deviations, there a few discrepancies between EW data and the SM predictions for quantities like $g_{\mu}-2$ or the left-right asymmetries in the hadronic and leptonic

\footnotetext{
${ }^{1}$ The fact that a Higgs doublet comes with an extra approximate global symmetry (the so called custodial symmetry), which automatically ensures that the $\rho$ parameter is equal to 1 , is certainly a welcome feature in this regards.
} 
sectors; (ii) the neutrino masses can be generated only if new states are added to the SM or if a new scale is introduced; (iii) the SM does not provide any dynamics to generate the observed matter-antimatter asymmetry; (iv) no SM particle can account for the dark matter (DM) relic abundance; (v) with may be the exception of the Higgs boson itself, no SM particle can drive inflation; (vi) there is no rationale for the pattern of fermion masses and mixing angles; (vii) the strong $\mathrm{CP}$ problem remains unexplained; (viii) the charge quantization most likely requires an embedding of the SM gauge group into a bigger symmetry which would unify all the fundamental interactions; (ix) gravity is left aside.

Theorists have always been very good at giving names to things they do not understand. And clearly the EWSB sector has been an inspirational source of creativity to them, as it is evident by collecting the attributes that have been associated to the Higgs boson over the last few years: burried, composite, fat, gauge, gaugephobic, intermediate, leptophilic, little, littlest, lone, phantom, portal, private, slim, simplest, twin, un- ... A description of these various constructions is certainly beyond the scope of this talk and I will limit myself to present a few examples that hopefully are representative of the possible structures governing the new physics needed around the Fermi scale.

\section{Supersymmetric Higgs(es)}

A tout seigneur, tout honneur: the supersymmetric (SUSY) extensions of the SM certainly stand out among the models of new physics for at least several reasons: (i) the absence of quadratic divergences; (ii) a dynamical EWSB driven radiatively by the top Yukawa interactions; (iii) the absence of large oblique corrections due to the $R$-parity that forbids any interactions between SM fields and an odd number of heavy fields; (iv) a precise apparent unification of the $S U(3) \times S U(2) \times$ $U(1)$ gauge couplings; (v) the possibility to identify the lightest supersymmetric particle (LSP) as the Dark Matter...

Moreover, SUSY models seems to be in even better agreement with EW data than the SM itself [4]. Unfortunately, this agreement is limited to small and peculiar regions of the (large) parameter space [5] and LEP2 data together with the lack of discovery of a Higgs boson below $114 \mathrm{GeV}$ or of any new states has forced supersymmetry into fine-tuning territory, partially undermining its original motivation. The most stringent constraint on the scale of SUSY breaking comes from the lower bound on the Higgs boson mass. Indeed at tree-level, the shape of the Higgs potential is dictated by gauge interactions:

$$
V=\left(|\mu|^{2}+m_{H_{u}}^{2}\right)\left|H_{u}^{0}\right|^{2}+\left(|\mu|^{2}+m_{H_{d}}^{2}\right)\left|H_{d}^{0}\right|^{2}-B\left(H_{u}^{0} H_{d}^{0}+\text { h.c. }\right)+\frac{g^{2}+g^{\prime 2}}{8}\left(\left|H_{u}^{0}\right|^{2}-\left|H_{d}^{0}\right|^{2}\right)^{2},
$$

and predicts a light $\mathrm{CP}$-even Higgs scalar $(\tan \beta$ measures the ratio of the two Higgs vacuum expectation values, $\left.\left\langle v_{u} / v_{d}\right\rangle\right)$ :

$$
m_{h}^{2}=m_{Z}^{2} \cos ^{2} 2 \beta
$$

One-loop radiative corrections can bring the Higgs mass above the LEP bound provided that a stop mass is heavier than about $1 \mathrm{TeV}$. But at the same time, the stop will also generate a correction to the $Z$ mass that should then be canceled by a large $\mu$ term. This cancelation requires a fine-tuning of the order of $1 \%$ (the amount of fine-tuning depends exponentially on the Higgs mass bound). This 
"SUSY little problem" has driven over the years a burst of activity in building concrete models allowing for a heavier Higgs and various interesting proposals have emerged, either with a low scale of SUSY breaking mediation [6] or with the addition of extra fields/symmetry/interactions to the minimal SUSY model (MSSM):

- more scalars: the NMSSM and its friends [7];

- more gauge fields (with new non-decoupled D-terms) [8];

- more global symmetry: such that the SUSY Higgses appear as Goldstone bosons [9];

- more interactions parametrized by higher dimensional terms: the BMSSM [10, 11]. This effective approach offers a model-independent look at the SUSY little hierarchy problem. At the lowest order, it has been shown [11] that the most general non-renormalizable interactions can be captured by the superpotential:

$$
W_{\mathrm{BMSSM}}=\frac{\lambda_{1}}{M}\left(H_{u} H_{d}\right)^{2}+\frac{\lambda_{2}}{M} \mathscr{Z}_{\text {soft }}\left(H_{u} H_{d}\right)^{2}
$$

with no correction to the Kähler potential. These two interactions certainly allow for an heavier Higgs and a much lighter stop [10, 11, 12], while keeping the EW vacuum (meta)stable [13]. At the same time, the window for MSSM baryogenesis is extended and more natural [14], while the LSP can account for DM relic abundance [15].

In the absence of any direct evidence for SUSY, it is important to keep an eye open on other possible alternatives that have emerged in the recent years, not necessarily aiming at providing a UV completion to the SM valid up to the Planck. All the models I will now present have an origin rooted in recent developments of string theory (branes, AdS/CFT correspondence) which has provided a new laboratory to address various particle physics questions [16].

\section{Little Higgs}

Symmetries of the EWSB sector can help to preserve the tree-level structure of the SM, i.e., can help to keep the oblique corrections under control. For instance, it is well-known that the embedding

$$
\frac{S U(2)_{L} \times U(1)_{Y}}{U(1)_{\mathrm{em}}} \subset \frac{S U(2)_{L} \times S U(2)_{R}}{S U(2)_{V}}=\frac{S O(4)}{S O(3)}
$$

is enough to ensure that the EWSB will not generate a contribution to the $T$ oblique parameter. The situation of the $S$ oblique parameter is notoriously more difficult to handle since the only symmetry that can protect it is the gauge $S U(2)_{L}$ symmetry itself ${ }^{2}$ and it has to be broken anyway. Therefore a contribution to $S$ is expected with a scaling like $v / \Lambda_{E W S B}$, where $v=246 \mathrm{GeV}$ is the SM Higgs v.e.v. and $\Lambda_{E W S B}$ is a typical mass scale of the EWSB sector. One way to reduce this contribution is to make $v$ much smaller than $\Lambda_{E W S B}$, which is another formulation of the gauge hierarchy problem.

\footnotetext{
${ }^{2}$ I thank Ben Gripaios for some valuable discussions on this issue.
} 
Again, enlarging the symmetry of the EWSB can provide a solution and ensure a naturally small contribution to the $S$ parameter: the embedding

$$
\frac{S O(4)}{S O(3)} \subset \frac{S O(5)}{S O(4)}, \frac{S U(5)}{S O(5)} \ldots
$$

allows to identify the full Higgs doublet as Nambu-Goldstone boson and leads to a naturally small ratio $v / \Lambda_{E W S B}$.

The idea behind the Little Higgs models [17, 18] is precisely to identify the Higgs doublet as a (pseudo) Nambu-Goldstone boson while keeping some sizable non-derivative interactions. By analogy with QCD where the pions $\pi^{ \pm, 0}$ appear as Nambu-Godstone bosons associated to the breaking of the chiral symmetry $S U(2)_{L} \times S U(2)_{R} / S U(2)_{\text {isospin }}$, switching on some interactions that break explicitly the global symmetry will generate a mass to the would-be massless NambuGoldstone bosons of the order of $g \Lambda_{G / H} /(4 \pi)$, where $g$ is the coupling of the symmetry breaking interaction and $\Lambda_{G / H}$ is the dynamical scale of the global symmetry breaking $G / H$. In the case of the Higgs boson, the top Yukawa interaction or the gauge interactions themselves will certainly break explicitly (part of) the global symmetry since they act non-linearly on the Higgs boson ${ }^{3}$. Therefore, obtaining a Higgs mass around $100 \mathrm{GeV}$ would demand a strong dynamical scale $\Lambda_{G / H}$ of the order of $1 \mathrm{TeV}$, which is known to lead to too large oblique corrections. Raising the strong dynamical scale by at least one order of magnitude requires an additional selection rule to ensure that a Higgs mass is generated at the 2-loop level only

$$
m_{h}^{2}=\frac{g^{2}}{16 \pi^{2}} \Lambda_{G / H}^{2} \rightarrow m_{h}^{2}=\frac{g_{1}^{2} g_{2}^{2}}{\left(16 \pi^{2}\right)^{2}} \Lambda_{G / H}^{2}
$$

The way to enforce this selection rule is through a "collective breaking" of the global symmetry:

$$
\mathscr{L}=\mathscr{L}_{G / H}+g_{1} \mathscr{L}_{1}+g_{2} \mathscr{L}_{2}
$$

Each interaction $\mathscr{L}_{1}$ or $\mathscr{L}_{2}$ individually preserves a subset of the global symmetry such that the Higgs remains an exact Nambu-Goldstone boson whenever either $g_{1}$ or $g_{2}$ is vanishing. A mass term for the Higgs boson can be generated by diagrams involving simultaneously both interactions only. At one-loop, there is not such a diagram that would be quadratically divergent. Explicitly, the cancellation of the SM quadratic divergences is achieved by a set of new particles around the Fermi scale: gauge bosons, vector-like quarks, and extra massive scalars, which are related to the SM particles by the original global symmetry. These new particles, with definite couplings to SM particles as dictated by the global symmetries of the theory, are perfect goals for the LHC.

The compatibility of Little Higgs models with experimental data is significantly improved when the global symmetry involves a custodial symmetry as well as a $T$-parity [20] under which, in analogy with $R$-parity in SUSY models, the SM particles are even and their partners are odd. Such Little Higgs models would therefore appear in colliders as jets with missing transverse energy [21]. There is also interesting physics associated to the partner(s) of the top quark which could be pairproduced by gluon fusion or single-produced by $W b$ busion, i.e., $W$-exchange in $t$-channel [22, 23].

\footnotetext{
${ }^{3}$ When part of the global symmetry is weakly gauged, the question of alignement of the gauge group with the unbroken global symmetry arises and can give non-trivial constraints on the parameter space of the models [19].
} 


\section{Strong vs. weak EWSB}

Supersymmetric models, Little Models and many other models assume that the answer to the pressing question of the origin of EWSB is already known: What is unitarizing the $W W$ scattering amplitude? I said earlier that the masses of the $W^{ \pm}$and $Z$ gauge bosons break the gauge symmetry. Actually, in presence of these masses, the gauge symmetry is realized non-linearly: the longitudinal $W_{L}^{ \pm}, Z_{L}$ can be described by the Nambu-Goldstone bosons, or pions, associated to the coset $S U(2)_{L} \times S U(2)_{R} / S U(2)_{\text {isospin }}$ and the gauge boson mass terms correspond to the pions kinetic term $\left(\sigma^{a}, a=1,2,3\right.$, are the usual Pauli matrices):

$$
\mathscr{L}_{\text {mass }}=\frac{v^{2}}{4} \operatorname{Tr}\left(D_{\mu} \Sigma^{\dagger} D^{\mu} \Sigma\right) \quad \text { with } \Sigma=e^{i \sigma^{a} \pi^{a} / v}
$$

Thanks to this Goldstone boson equivalence [24], the non-trivial scattering of the longitudinal $W$ 's ( $W$ generically denotes $W^{ \pm}$as well as $Z$ ) now simply follows for the contact interactions among four pions obtained by expanding the Lagrangian (5.1) and leads to amplitudes that grow with the energy:

$$
\mathscr{A}\left(W_{L}^{a} W_{L}^{b} \rightarrow W_{L}^{c} W_{L}^{d}\right)=\mathscr{A}(s) \delta^{a b} \delta^{c d}+\mathscr{A}(t) \delta^{a c} \delta^{b d}+\mathscr{A}(u) \delta^{a d} \delta^{b c} \text { with } \mathscr{A}(s) \approx \frac{s}{v^{2}} .
$$

In the absence of any new weakly coupled elementary degrees of freedom canceling this growth, perturbative unitarity will be lost around ${ }^{4} 1.2 \mathrm{TeV}$ and new strong dynamics will kick in and soften the UV behavior of the amplitude, for instance via the exchange of massive bound states similar to the $\rho$ meson of QCD. In any circumstances, by measuring the $W^{ \pm}$and $Z$ masses, we have been guaranteed to find new physics around the Fermi scale to ensure the proper decoupling of the longitudinal polarizations at very high energy.

The simplest example of new dynamics that can restore perturbative unitarity consists of a single scalar field, $h$, singlet under $S U(2)_{L} \times S U(2)_{R}$ and coupled to the longitudinal $W$ 's as [25]:

$$
\mathscr{L}_{\mathrm{EWSB}}=\frac{1}{2} \partial_{\mu} h \partial^{\mu} h-V(h)+\frac{v^{2}}{4} \operatorname{Tr}\left(D_{\mu} \Sigma^{\dagger} D^{\mu} \Sigma\right) \times\left(1+2 a \frac{h}{v}+b \frac{h^{2}}{v^{2}}\right) .
$$

Via its linear coupling, $a$, to the $W_{L}$ 's, the scalar gives an additional contribution to the $W W$ scattering amplitude

$$
\mathscr{A}_{\text {scalar exchange }}(s)=-\frac{a^{2} s^{2}}{v^{2}\left(s-m_{h}^{2}\right)},
$$

which, for $a=1$, cancels the leading contact term at high energy. This is not the end of the story yet: perturbative unitarity should also be maintained in inelastic channels too, like $W_{L} W_{L} \rightarrow h h$. Both the linear and quadratic couplings, $a$ and $b$, contribute to this amplitude and the terms growing with the energy are canceled for the particular choice $b=a^{2}$. The point $a=b=1$ defines the SM Higgs boson and it can be shown that the scalar resonance and the pions then combine together to form a doublet transforming linearly under $S U(2)_{L} \times S U(2)_{R}$.

\footnotetext{
${ }^{4}$ Defining the breakdown of perturbativity is subject to arbitrary choices: the $1.2 \mathrm{TeV}(=2 \sqrt{2 \pi} v)$ number follows from requiring that the real part of the partial waves of the iso-amplitudes remains smaller than $1 / 2$, while demanding that the tree-level amplitude remains bigger than the one-loop one leads to the more conventional scale, $4 \pi v(\approx 3.1 \mathrm{TeV})$, associated to a non-linear $\sigma$-model with a breaking scale $v$.
} 
The Lagrangian (5.3) describes either an elementary Higgs boson or a composite one emerging as a bound state of a strongly interacting sector. In the latter case, we do expect the couplings to deviate from $a=b=1$ (I will show in Section 6 that these deviations scale are controlled by the ratio of the weak scale over the Higgs compositeness scale, $f$ ) and therefore the Higgs boson alone fails to fully unitarize the $W W$ scattering amplitude.
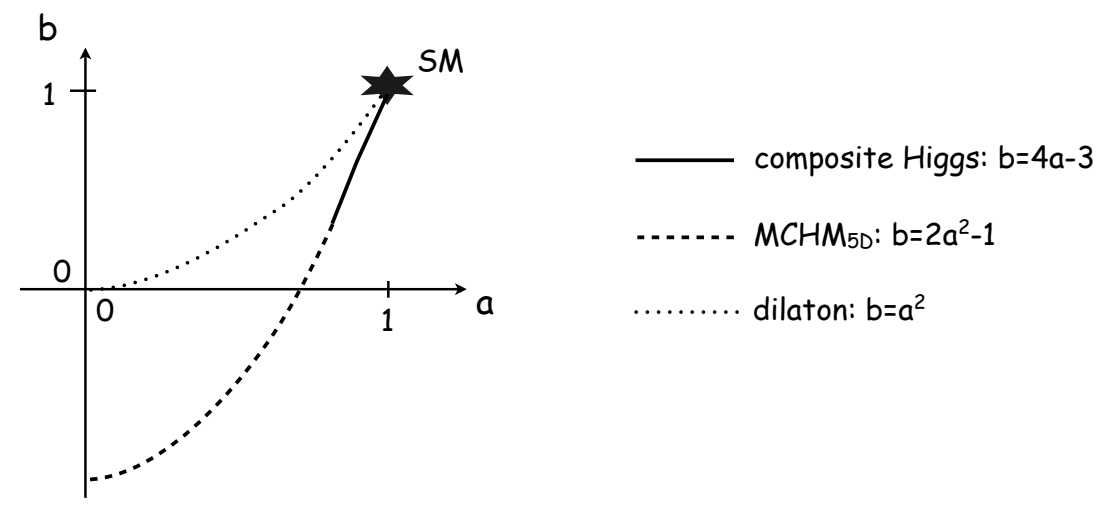

Figure 1: (Partial) unitarization of scattering amplitudes with a scalar field. $a$ and $b$ are the linear and quadratic couplings of the scalar to the gauge bosons defined in Eq. (5.3). The particular point $a=b=1$ corresponds to the SM and neither the elastic nor the inelastic scattering amplitudes are growing with the energy. Composite Higgs models with a rather large compositeness scale, $f \gg v$, are seating on an universal line away from the SM (this universal behavior is the result of the fact that the scattering amplitudes are dominated by a single operator, as I will show in Section 6). When the compositeness scale is lowered towards the weak scale, the series in $v / f$ has to be resumed and model dependent effects appear. For instance, the minimal composite Higgs model $\left(\mathrm{MCHM}_{5 D}\right)$ of Ref. [26] seats along the curve $b=2 a^{2}-1$. It was shown in Ref. [27] that, under very generic assumptions, in composite Higgs models, $a$ is always smaller than 1 . The dilaton couplings can also be described by the Lagrangian (5.3) and they satisfy $b=a^{2}$, therefore the double dilaton production never becomes strong.

Composite Higgs models are examples of models where the breakdown of perturbative unitarity is postponed to higher energy ${ }^{5}$. In the next section, I will discuss in details the collider signatures of these models. The extra dimensional Higgsless models that will be presented in Section 7 are other examples with delayed perturbative unitarity breakdown thanks to a non-trivial dynamics of a tower of spin 1 massive particles. Both classes of models where already considered in the eighties, see for instance Ref. [29] for a historical perspective, but they have experienced a recent revival and can now be cast in terms of models with warped extra dimensions.

\section{Composite Higgs models}

Notwithstanding its simplicity, the appeal of the SM Higgs picture comes from its successful agreement with EW precision data, provided that the Higgs boson is rather light. To this regards,

\footnotetext{
${ }^{5} \mathrm{~A}$ famous theorem due to Cornwall et al. [28] states that the only way to fully ensure perturbative unitarity in all possible elastic and inelastic channels is via the exchange a Higgs boson in a spontaneously broken gauge theory. We explore here the possibility to delay the perturbative unitarity breakdown.
} 
being an elementary scalar is not a virtue but rather a flaw especially when facing radiative corrections. It thus tantalizing to consider the Higgs boson as a composite bound state emerging from a strongly-interacting sector. In order to maintain a good agreement with EW data, it is sufficient that a mass gap separates the Higgs resonance from the other resonances of strong sector (the resonances that will ultimately enforce a good behavior of the $W W$ scattering amplitudes). Such a mass gap can naturally follows from dynamics if the strongly-interacting sector possesses a global symmetry, $G$, spontaneously broken at a scale $f$ to a subgroup $H$, such that the coset $G / H$ contains a fourth Nambu-Goldstone bosons that can be identified with the Higgs boson. Simple examples of such coset are $S U(3) / S U(2)$ or $S O(5) / S O(4)$, the latter being favored since it is invariant under the custodial symmetry. Attempts to construct composite Higgs models in $4 \mathrm{D}$ have been made by Georgi and Kaplan (see for instance Ref. [30]) and modern incarnations have been recently investigated in the framework of 5D warped models where, according to the principles of the AdS/CFT correspondence, the holographic composite Higgs boson now originates from a component of a gauge field along the 5th dimension with appropriate boundary conditions.

The composite Higgs models offer a nice and continuous interpolation between the SM and technicolor type models. The dynamical scale $f$ defines the compositeness scale of the Higgs boson: when $\xi=v^{2} / f^{2} \rightarrow 1$, the Higgs boson appears essentially as a light elementary particle (and its couplings approach the ones predicted by the SM) while the other resonances of the strong sector become heavier and heavier and decouple; on the other hand, when $\xi \rightarrow 1$, the couplings of the Higgs boson to the $W_{L}$ 's go to zero and unitarity in gauge boson scattering is ensured by the exchange of the heavy resoances.

At the eve of the LHC operation, I would like to give a description of the physics of such a composite Higgs boson rather than presenting the details of the construction of an explicit model. In the same way that we do not need of the refinements of QCD to describe the physics of the pions, I will rely on an effective Lagrangian to capture the relevant physics. This effective Lagrangian involves higher dimensional operators for the low energy degrees of freedom (the SM particles and a unique Higgs boson in the minimal case) and the strong sector will be broadly parametrized by two quantities: the typical mass scale, $m_{\rho}$, of the heavy resonances and the dynamical scale, $f$, associated to the coset $G / H$ (for maximally strongly coupled sectors, we expect $m_{\rho} \approx 4 \pi f$; here, I will simply assume that $m_{\rho}$ is parametrically large than $f$ ). There are two classes of higher dimensional operators: (i) those that are genuinely sensitive to the new strong force and will affect qualitatively the physics of the Higgs boson and (ii) those that are sensitive to the spectrum of the resonances only and will simply act as form factors. Simple rules control the size of these different operators, see Ref. [31], and the effective Lagrangian generically takes the form ${ }^{6}$ :

$$
\begin{aligned}
& \mathscr{L}_{\text {SILH }}=\frac{c_{H}}{2 f^{2}}\left(\partial_{\mu}\left(H^{\dagger} H\right)\right)^{2}+\frac{c_{T}}{2 f^{2}}\left(H^{\dagger} \overleftrightarrow{D}{ }_{\mu} H\right)^{2}-\frac{c_{6} \lambda}{f^{2}}\left(H^{\dagger} H\right)^{3}+\left(\frac{c_{y} y_{f}}{f^{2}} H^{\dagger} H \bar{f}_{L} H f_{R}+\text { h.c. }\right) \\
& +\frac{i c_{W} g}{2 m_{\rho}^{2}}\left(H^{\dagger} \sigma^{i} \overleftrightarrow{D^{\mu}} H\right)\left(D^{v} W_{\mu v}\right)^{i}+\frac{i c_{B} g^{\prime}}{2 m_{\rho}^{2}}\left(H^{\dagger} \overleftrightarrow{D^{\mu}} H\right)\left(\partial^{v} B_{\mu v}\right)+\ldots
\end{aligned}
$$

Some oblique corrections are generated, at tree-level, by the operators of this effective Lagrangian: (i) the operator $c_{T}$ gives a contribution to the $T$ Peskin-Takeuchi parameter, $\hat{T}=c_{T} v^{2} / f^{2}$,

\footnotetext{
${ }^{6} g, g^{\prime}$ are the SM EW gauge couplings, $\lambda$ is the SM Higgs quartic coupling and $y_{f}$ is the SM Yukawa coupling to the fermion $f_{L, R}$. All the coefficients, $c_{H}, c_{T} \ldots$, appearing in Eq. (6.1) are expected to be of order one.
} 
which would impose a very large compositeness scale; however, assuming that the custodial symmetry is preserved by the strong sector, the coefficient of this operator is vanishing automatically; (ii) a contribution to the $S$ parameter is generated by the form factor operators only, $\hat{S}=$ $\left(c_{W}+c_{B}\right) m_{W}^{2} / m_{\rho}^{2}$, and will simply impose a lower bound on the mass of the heavy resonances, $m_{\rho} \geq 2.5 \mathrm{TeV}$. At the loop level, the situation is getting a bit more complicated: as I am going to show below, the couplings of the Higgs to the SM vectors receive some corrections of the order $v^{2} / f^{2}$, and these corrections prevent the nice cancelation occurring in the SM between the Higgs and the gauge boson contributions and $S$ and $T$ are logarithmically divergent [32]. Typically, this one-loop IR contribution imposes $f^{2} / v^{2} \geq 2 \div 3$.

One may worry that because of the modified Yukawa interactions induced by the operator $c_{y}$, the mass matrices and the Yukawa interaction matrices are not simultaneously diagonalizable, leading to potentially dangerous flavor changing neutral currents (FCNC). Actually, the operator $c_{y}$ is flavor universal (at least among the light fermions) and no Higgs-mediated FCNC is generated at the leading level (see however Refs. [33, 34] for a detailed discussion on this subject).

The effective Lagrangian (6.1) does induce some corrections to the Higgs couplings to the SM particles. In particular, the operator $c_{H}$ gives a correction to the Higgs kinetic term which can be brought back to its canonical form at the price of a proper rescaling of the Higgs field inducing an universal shift of the Higgs couplings by a factor $1-c_{H} v^{2} /\left(2 f^{2}\right)$. For the fermions, this universal shift adds up to the modification of the Yukawa interactions:

$$
\begin{aligned}
& g_{h f \bar{f}}^{\xi}=g_{h f \bar{f}}^{\mathrm{SM}} \times\left(1-\left(c_{y}+c_{H} / 2\right) v^{2} / f^{2}\right), \\
& g_{h W W}^{\xi}=g_{h W W}^{\mathrm{SM}} \times\left(1-c_{H} v^{2} /\left(2 f^{2}\right)\right) .
\end{aligned}
$$

All the dominant corrections, i.e. the ones controlled by the strong operators, preserve the Lorentz structure of the SM interactions, while the form factor operators will also introduce couplings with a different Lorentz structure.

The effective Lagrangian (6.1) should be viewed as the first terms in an expansion in $\xi=$ $v^{2} / f^{2}$. When departing significantly from the SM limit, $v^{2} / f^{2} \sim \mathscr{O}(1)$, the series has to be resummed. Explicit models, like the ones constructed in 5D warped space [26], provide examples of such a resummation, allowing to study the effects of the anomalous Higgs couplings up to the technicolor limit. Figure 2 shows the modification in the branching ratios for the Higgs decays to SM particles in the minimal composite Higgs model with fermions embedded into fundamental representations of $S O(5)$.

The Higgs anomalous couplings affect the decay rates as well as the production cross sections of the Higgs. Therefore, the searches for the Higgs boson at the LHC, as well as the LEP/Tevatron exclusion bounds are modified as compared to the SM case. Figure 3 reports the amount of luminosity needed for discovery in the most promising channels for the minimal composite Higgs models of Ref. [26].

Will the LHC be able to probe these deviations in the couplings ${ }^{7}$ of the Higgs? The contribution of the operator $c_{H}$ is universal for all Higgs couplings and therefore it does not affect the Higgs

\footnotetext{
${ }^{7}$ The physics of the composite models, as captured by the effective Lagrangian (6.1), selects the operators $c_{H}$ and $c_{y}$ as the most important ones for LHC studies, as opposed to totally model-independent operator analyses [37, 38] which often lead to the conclusion that the dominant effects should appear in the vertices $h \gamma \gamma$ and $h g g$, since their SM contribution occurs only at loop level.
} 

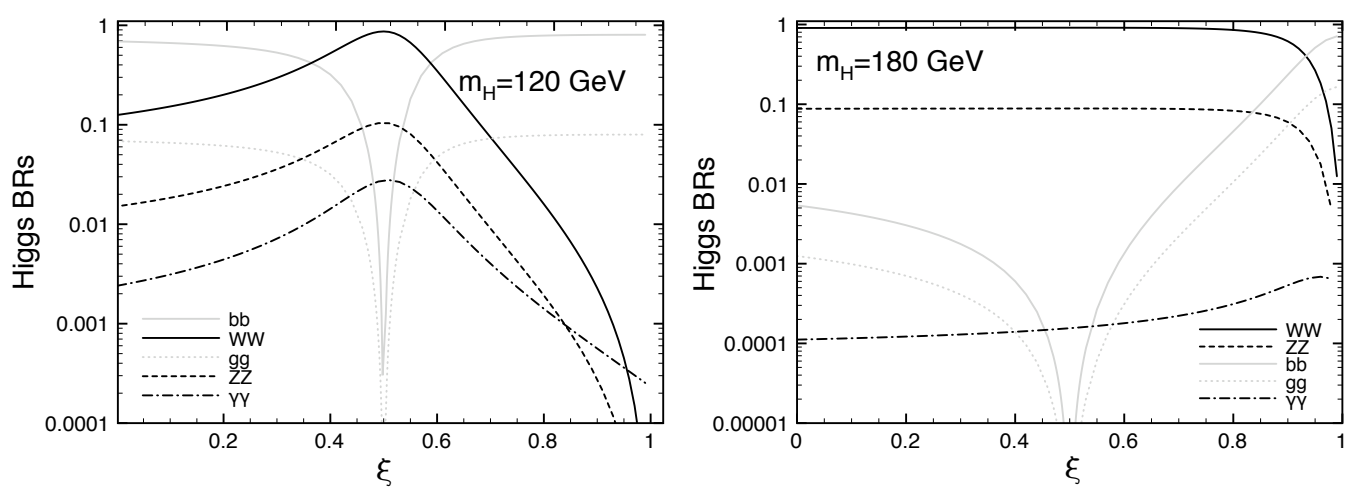

Figure 2: Higgs decay branching ratios as a function of $\xi=v^{2} / f^{2}$ for SM fermions embedded into fundamental representations of $S O(5)$ for two benchmark Higgs masses: $m_{h}=120 \mathrm{GeV}$ (left plot) and $m_{h}=180 \mathrm{GeV}$ (right plot). For $\xi=0.5$, the Higgs is fermiophobic, while in the technicolor limit, $\xi \rightarrow 1$, the Higgs becomes gaugephobic. From Ref. [25].

branching ratios, but only the total decay width and the production cross section. The measure of the Higgs decay width at the LHC is very difficult and it can be reasonably done only for rather heavy Higgs bosons, well above the two gauge boson threshold, a region which is not of particular interest since we consider the Higgs as a pseudo-Goldstone boson, and therefore relatively light. However, for a light Higgs, LHC experiments can measure the product $\sigma_{h} \times B R_{h}$ in many different channels: production through gluon, gauge-boson fusion, and top-strahlung; decay into $b, \tau, \gamma$ and (virtual) weak gauge bosons. At the LHC with about $300 \mathrm{fb}^{-1}$, it is possible to measure Higgs production rate times branching ratio in the various channels with $20-40 \%$ precision $[39,40]$. For $c_{H}$ and $c_{y}$ of order one, this will translate into a sensitivity on the compositeness scale of the Higgs, $4 \pi f$, up to $5 \div 7 \mathrm{TeV}$.

Cleaner experimental information can be extracted from ratios between the rates of processes with the same Higgs production mechanism, but different decay modes. In measurements of these ratios of decay rates, many systematic uncertainties drop out. However, the Higgs coupling determinations at the LHC will still be limited by statistics, and therefore they can benefit from a luminosity upgrade, like the sLHC. At a linear collider, like a ILC operating at $\sqrt{s}=500 \mathrm{GeV}$ [41], precisions on $\sigma_{h} \times B R_{h}$ can reach the percent level [42], providing a very sensitive probe on the compositeness scale of the Higgs up to $4 \pi f \sim 30 \mathrm{TeV}$. Moreover, a linear collider can test the existence of the operator $c_{6}$ that controls the Higgs self interactions, since the triple Higgs coupling can be measured with an accuracy of about $10 \%$ for $\sqrt{s}=500 \mathrm{GeV}$ and an integrated luminosity of $1 \mathrm{ab}^{-1}$ [44]. CLIC running at $\sqrt{s}=3 \mathrm{TeV}$ [], will improve these sensitivities by a factor 2 .

Deviations from the SM predictions of Higgs production and decay rates could be a hint towards models with strong dynamics, especially if no new light particles are discovered at the LHC. However, they do not unambiguously imply the existence of a new strong interaction. The most characteristic signals of a composite Higgs model have to be found in the very high-energy regime. Indeed, as already announced in Section 5, a peculiarity of a composite Higgs boson is that it fails to fully unitarize the $W_{L} W_{L}$ scattering amplitudes which have thus a residual growth with energy and the corresponding interaction becomes strong, eventually violating tree-level unitarity at the 

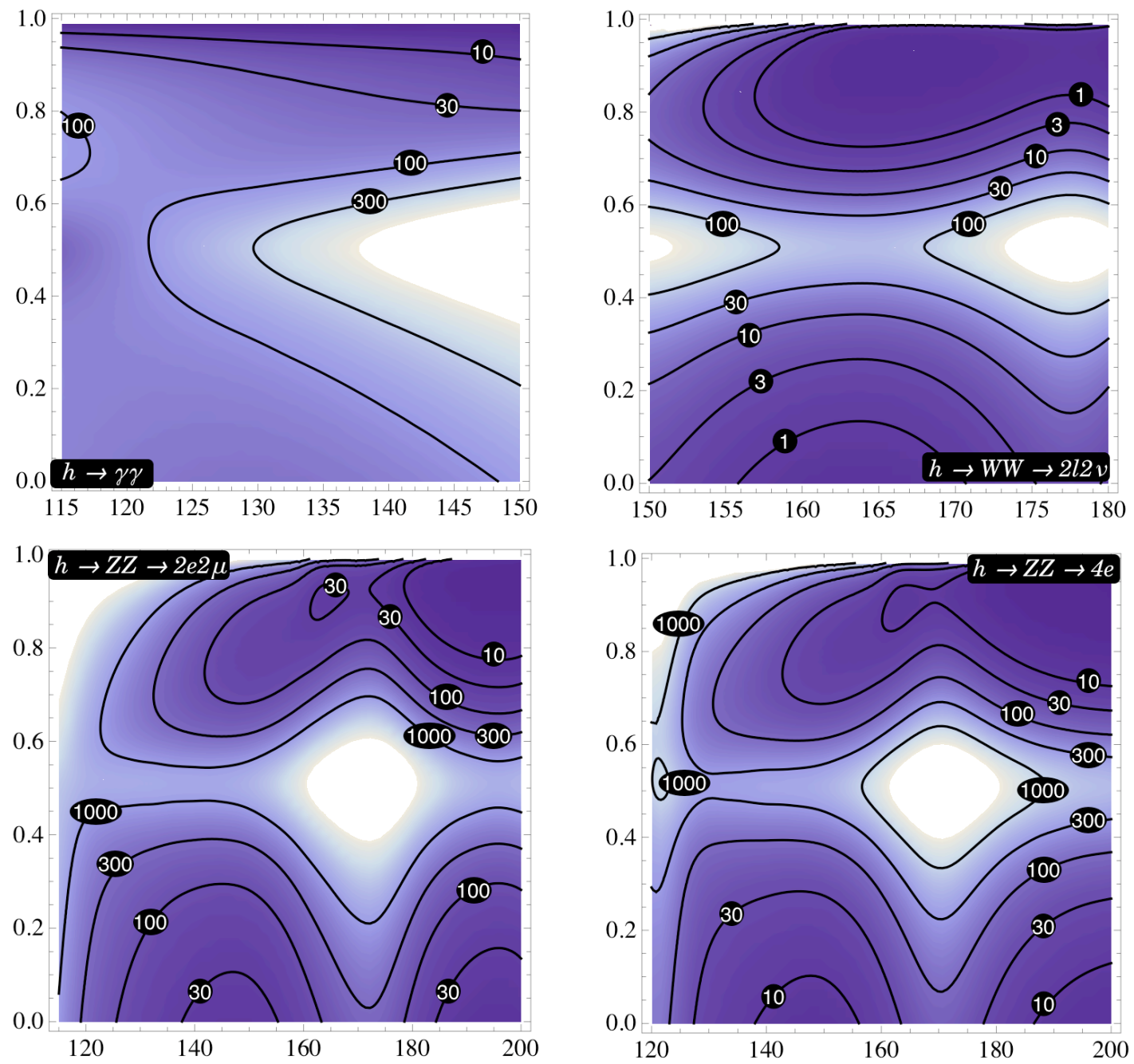

Figure 3: Luminosity needed for discovery in the most promising channels with the CMS detector [35] as a function of the Higgs mass and the parameter, $\xi=v^{2} / f^{2}$, measuring the amount of compositeness of the Higgs boson. These contour plots correspond to the minimal composite Higgs models of Ref. [26]. For $x i=0.5$, the Higgs boson becomes leptophilic and thus harder to detect. From Ref. [36].

cutoff scale. Indeed, the extra contribution to the Higgs kinetic term from the $c_{H}$ operator prevents Higgs exchange diagrams from accomplishing the exact cancellation, present in the SM, of the terms growing with energy in the amplitudes. Therefore, although the Higgs is light, we obtain strong $W W$ scattering at high energies.

From the operator $c_{H}$, using the Goldstone equivalence theorem, it is easy to derive the following high-energy limit of the scattering amplitudes for longitudinal gauge bosons

$$
\mathscr{A}\left(W_{L}^{a} W_{L}^{b} \rightarrow W_{L}^{c} W_{L}^{d}\right)=\mathscr{A}(s) \delta^{a b} \delta^{c d}+\mathscr{A}(t) \delta^{a c} \delta^{b d}+\mathscr{A}(u) \delta^{a d} \delta^{b c} \quad \text { with } \mathscr{A}(s) \approx \frac{s}{f^{2}} .
$$

This result is correct to leading order in $s / f^{2}$, and to all orders in $v^{2} / f^{2}$ in the limit $g_{\mathrm{SM}}=0$, when the $\sigma$-model is exact. The growth with energy of the amplitudes is strictly valid only up 

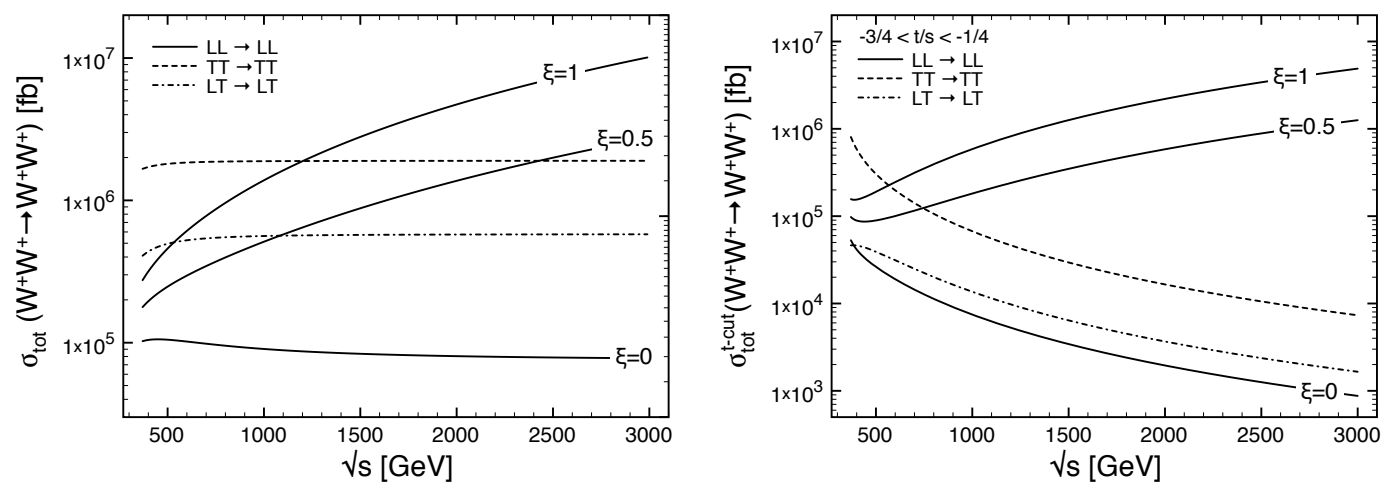

Figure 4: $W^{+} W^{+} \rightarrow W^{+} W^{+}$partonic cross section as a function of the center of mass energy for $m_{h}=$ $180 \mathrm{GeV}$ for the SM $(\xi=0)$ and for composite Higgs models $\left(\xi=v^{2} / f^{2} \neq 0\right)$. On the left, the inclusive cross section (with a cut on $t$ and $u$ of the order of the $W$ squared mass) is shown. The right plot presents the hard cross section with a cut $-0.75<t / s<-0.25$. Within the SM, the scattering cross section of the longitudinal polarizations is over-dominated by the one of the transverse polarizations. Therefore the onset of strong scattering in composite Higgs models is delayed to energies much higher than $M_{W}$, as estimated by NDA. The enhancement of the amplitude for the transverse polarization is due to the structure of the Coulomb terms for the exchange of the photon and the $Z$ in the $t$ - and $u$-channels. From Ref. [25].

to the maximum energy of our effective theory, namely $m_{\rho}$. The behaviour above $m_{\rho}$ depends on the specific model realization. In 5D models, the growth of the elastic amplitude is softened by Kaluza-Klein modes exchange [45], but the inelastic channel dominate and strong coupling is reached at a scale $\sim 4 \pi f$. Notice that the amplitudes (6.4) are exactly proportional to the scattering amplitudes obtained in a Higgsless SM, the growth being controlled by the strong coupling scale, $f$, and not the weak scale itself, $v$.

Will the LHC be able to measure the growth of these scattering amplitudes? Contrary to a naive belief, It is a notoriously difficult measurement which requires some large integrated luminosity [46]. The most promising channels correspond to purely leptonic decays of the $W$ 's, though semileptonic decay channels have also been considered recently [47, 48]. The rapid falloff of the $W$ luminosity inside the proton and the numerous SM background that can fake the signal certainly make the measurement harder, but, as a matter of fact, already at the partonic level, the onset of the strong scattering is delayed to higher energies due to a large pollution from the scattering of the transverse polarizations [25], as illustrated in Fig. 4.

In composite Higgs models, another direct probe of the strong dynamics at the origin of EWSB is the cross section for the double Higgs production. Indeed, the Higgs boson appears as a pseudo Nambu-Goldstone boson and its properties are directly related to those of the other exact (eaten) Goldstones, corresponding to the longitudinal $W, Z$ gauge bosons. Thus, a generic prediction is that the strong gauge boson scattering is accompanied by strong production of Higgs pairs. The amplitudes for double Higgs production grow with the center-of-mass energy as

$$
\mathscr{A}\left(Z_{L}^{0} Z_{L}^{0} \rightarrow h h\right)=\mathscr{A}\left(W_{L}^{+} W_{L}^{-} \rightarrow h h\right)=\frac{c_{H} s}{f^{2}} .
$$

Therefore a significant enhancement over the (negligible) SM rate for the production of two Higgs 
bosons at high $p_{T}$, along with two forward jets associated with the two primary partons that radiated the $W_{L} W_{L}$ pair, is expected. An explorative analysis [25] has shown that the best channel for discovery involves 3 leptons in the final states, with both Higgs bosons decaying to $W^{+} W^{-}$: $p p \rightarrow h h j j \rightarrow 4 W j j \rightarrow l^{+} l^{-} l^{ \pm} \mathscr{H}_{\mathrm{T}} 4 j$. The final states are undeniably more complicated than in the analyses of gauge boson scattering and come with smaller branching ratios, but at least the double Higgs production does not suffer from pollution from the transverse modes and it it the only process that gives access to the quadratic coupling $b$ of the Lagrangian (5.3) and allows to test its relation to the linear coupling, $a$, as predicted by the structure of the higher dimension operators (6.1): $a=1-c_{H} v^{2} /\left(2 f^{2}\right), b=1-2 c_{H} v^{2} / f^{2}$. A Monte-Carlo simulation with simple kinematic cuts concludes [25] that the signal significance at the LHC operating at $\sqrt{s}=14 \mathrm{TeV}$ with $300 \mathrm{fb}^{-1}$ will be limited to about 2.5 standard deviations for $v^{2} / f^{2}=0.8$. With an upgrade of the LHC luminosity (sLHC program), a $5 \sigma$ discovery can be reached with less than $1 \mathrm{ab}^{-1}$ of integrated luminosity.

In conclusion, in the plausible situation that the LHC sees a Higgs boson and no other direct evidence of new physics, it will not be immediate to determine the true nature of this Higgs boson and tell for sure if it is an elementary particle or a composite bound state emerging from a strongly interacting sector. In that situation, a physics case for a linear collider together with the sLHC can be easily made.

\section{5D Higgsless models}

In composite models, when the compositeness scale gets close to the weak scale, the Higgs boson effectively decouples. This Higgsless limit is easily reached in 5D dimensional setups and offers a new point of view on the mass problem. In a sense, the EWSB itself is achieved via boundary conditions (rather than by a Higgs vacuum expectation value). According to the Einstein's relation between the mass and the momentum ( $\vec{p}_{3}$ denotes the momentum along the usual 3 spatial dimension and $p_{\perp}$ is the momentum along the extra dimension):

$$
m^{2}=E^{2}-\vec{p}_{3}^{2}-p_{\perp}^{2},
$$

a transverse momentum, $p_{\perp}$, simply appears as a mass from the 4D point of view. And the mass problem reduces to a problem of quantum mechanics in a box: suitable boundary conditions will generate a transverse momentum for the appropriate particles. Nonetheless, an immediate question arises: is it better to generate a transverse momentum than to introduce by hand a symmetry breaking mass for the gauge field? In other words, how is unitarity restored? In full generality, the elastic scattering amplitude of a massive Kaluza-Klein (KK) gauge field would have terms that grow with the fourth and the second powers of the energy

$$
\mathscr{A}=\mathscr{A}^{(4)}\left(\frac{E}{M}\right)^{4}+\mathscr{A}^{(2)}\left(\frac{E}{M}\right)^{2}+\mathscr{A}^{(0)}+\ldots
$$

In the SM, $\mathscr{A}^{(4)}$ is automatically vanishing due to gauge invariance, while $\mathscr{A}^{(2)}$ vanishes via the exchange of the physical Higgs boson. In 5D Higgsless models, the unitarization of the $W W$ scattering amplitudes follows from the exchange of all the KK excitations of the $W$. In order for this unitarization to actually happen, the couplings and the masses of the KK excitations have to 
obey the following sum rules ${ }^{8}[49]$ :

$$
\begin{gathered}
g_{W W W W}^{2}=g_{W W \gamma}^{2}+g_{W W Z}^{2}+\sum_{n} g_{W W Z^{(n)}}^{2} \\
4 g_{W W W W}^{2} M_{W}^{2}=3 g_{W W Z}^{2} M_{Z}^{2}+3 \sum_{n} g_{W W Z^{(n)}}^{2} M_{Z^{(n)}}^{2} .
\end{gathered}
$$

The effective couplings among the KK states are dictated by the gauge structure of the 5D theory and it is easy to show that the two sum rules are automatically satisfied, provided there is no hard breaking of gauge invariance, i.e., if the 5D gauge fields obey Dirichlet or Neumann boundary conditions at the end points of the $5^{\text {th }}$ dimension.

Is this KK unitarization a counter example of the theorem by Cornwall et al. mentioned in Section 5? Not exactly since, as the energy grows, more and more inelastic channels open up and they ultimately saturate the perturbative unitarity bound [50,51] around the scale $4 \pi^{4} M_{W}^{2} /\left(g^{2} M_{Z^{(1)}}\right)$ ( $g$ is the usual $S U(2)$ gauge coupling). Thus, we can see that for this scale to be substantially above the usual unitarity violation scale of the SM without a Higgs, one needs to have the first $\mathrm{KK}$ resonance to be as light as possible. The existence of a $W^{\prime}$ and a $Z^{\prime}$ below $1 \mathrm{TeV}$ and with significant cubic couplings to the SM gauge bosons is a robust prediction of Higgsless models.

For a concrete implementation of the Higgsless idea, one can conveniently use a warped extra dimension [52] ${ }^{9}$, which (i) automatically ensures a mass gap between the $W, Z$ and their KK excitations and (ii) is instrumental to enforce a custodial $S U(2)$ symmetry that is implemented as a bulk $S U(2)_{L} \times S U(2)_{R} \times U(1)_{B-L}$ gauge symmetry. The way the proper symmetry breaking is achieved is, see Fig. 5, by breaking the bulk gauge group down to the SM group $S U(2)_{L} \times U(1)_{Y}$ on the UV brane, thus ensuring that the additional gauge symmetry only manifests itself as a global symmetry among the KK states. The electroweak symmetry breaking is then achieved by breaking $S U(2)_{L} \times S U(2)_{R}$ to $S U(2)_{D}$ on the IR brane, which, according to the AdS/CFT principles, appears as a spontaneous breaking of the effective theory.

In the SM, the physical Higgs boson is instrumental in bringing the theory is good agreement with EW data. In the 5D Higgsless model described above, the first corrections to the EW precision observables are given by $[55,56]$

$$
S \equiv \frac{6 \pi}{g^{2} \log R^{\prime} / R} \sim 1.15, T \equiv 0 .
$$

Thus, while the $T$ parameter is protected by the built-in custodial symmetry, the $S$ paremeter is too large. Nonetheless, $S$ can be tuned away by delocalizing the (light) fermions in the bulk [57, 58]. For a particular profile of the fermion wavefunctions, the fermions will almost decouple from the gauge boson KK excitations and the resulting $S$ parameter will vanish. However, some doubts about the radiative stability of this delocalization setup have been raised [59]. Furthermore, delocalizing the fermions in the bulk also precludes a nice dynamical origin of the fermion mass hierarchy and the model has to be supplemented with an additional flavor structure (see for instance Ref. [60]).

\footnotetext{
${ }^{8} g_{W W W W}^{2}$ is the quartic $W$ self-coupling, $g_{W W X}$ is the cubic coupling between two $W$ 's and $X$ and $Z^{(n)}$ denote the KK excitations of the $Z$. The two sum rules (7.3) correspond to the $W^{ \pm} W^{ \pm} \rightarrow W^{ \pm} W^{ \pm}$channel and similar sum rules will apply to other $W Z$ channels.

${ }^{9}$ Alternatively, deconstruction versions have been proposed (see for instance Ref. [55] and references therein), which extend the pioneering BESS model [54]
} 


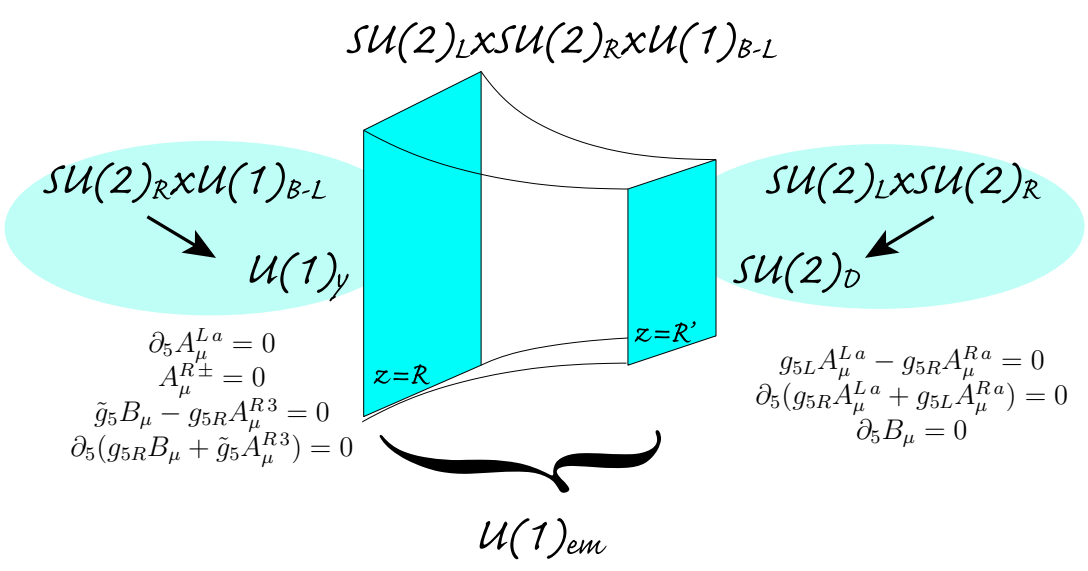

Figure 5: The symmetry-breaking structure of the warped Higgsless model [52]. The model considers a 5D gauge theory in a fixed gravitational anti-de-Sitter (AdS) background. The UV brane (sometimes called the Planck brane) is located at $z=R$ and the IR brane (also called the TeV brane) is located at $z=R^{\prime} . R$ is the AdS curvature scale. In conformal coordinates, the AdS metric is given by $d s^{2}=(R / z)^{2}\left(\eta_{\mu \nu} d x^{\mu} d x^{\nu}-d z^{2}\right)$.

The remaining problem with precision EW data is the compatibility of a large top quark mass with the observed $Z b_{L} \bar{b}_{L}$ coupling. The large top mass requires both the left-handed and righthanded top quarks to have a profile which is localized toward the IR brane. However, if the lefthanded top (and thus the left-handed bottom) are too close to the IR brane, then the gauge couplings of the bottom will be too different from the down and strange quarks. This problem may be avoided by separating the physics which generates the top quark mass [61], by allowing a Higgs vacuum expectation value to extend slightly into the bulk [62] or by introducing new fermionic states with exotic charges $[63,64]$.

The most distinctive feature of the Higgsless models is, of course, the absence of a physical scalar state in the spectrum. Yet, the absence of proof is not the proof of the absence and some other models exist in which the Higgs is unobservable at the LHC (for a recent review, see for instance Ref. [65]). Fortunately, Higgsless models are characterized by other distinctive features, such as (i) the presence of spin-1 KK resonances with the $W, Z$ quantum numbers, (ii) some slight deviations in the universality of the light fermion couplings to the SM gauge bosons and (iii) some deviations in the gauge boson self-interactions compared with the SM. References [66, 67] studied the production of the lightest $\mathrm{KK}$ excitations of the $W$ and the $Z$ via vector boson fusion. The most recent study [68] included also the more model-dependent possibility of Drell-Yan production. At the LHC, about $10 \mathrm{fb}^{-1}$ of luminosity will be necessary for the discovery of the resonances in the $700 \mathrm{GeV}$ mass range. A precise measurement of the couplings of these resonances or the search for some deviations in the SM couplings will require a more precise machine, such as an ILC or CLIC.

\section{Conclusion}

The SM has emerged as a successful description, at the quantum level, of the interactions 
among the elementary particles but it is at odds in what concerns the masses of these elementary particles. EW interactions certainly need three Nambu-Goldstone bosons to provide a mass to the $W^{ \pm}$and the $Z$ gauge bosons. But they also need new dynamics to act as a UV moderator and ensure a proper decoupling at high energy of the extra polarizations associated to the mass of these spin-1 fields. After many years of theoretical speculations and in the absence of any direct experimental evidences, it is not yet possible to tell whether the strength of this new dynamics is weak or strong. In many regards, this question is equivalent to asking whether a light Higgs boson exists or not. However it is also possible and plausible that a light and narrow Higgs-like scalar does exist but it is actually a bound state from some strong dynamics not much above the weak scale. Such composite models provide a continuous dynamical deformation of the SM.

The LHC is prepared to discover the Higgs boson or whatever replaces it. To this end, the collaboration between experimentalists and theorists is more important than ever to make sure, for instance, that no unexpected physics is missed because of triggers and cuts. In this regards, signature-motivated approaches like 'unparticles', 'hidden valleys' or 'quirks' should be encouraged.

Finally, it should not be forgotten that the LHC will be a top and a top-quark machine. And there are many reasons to believe that the top quark can be an important agent in the dynamics triggering the electroweak symmetry breaking. More than ever, experimental data are eagerly awaited to disentangle what may be the most pressing question faced by particle physics today: How is electroweak symmetry broken?

\section{Acknowledgements}

It is pleasure to thank my various collaborators, especially C. Csáki and J. Terning for fruitful works on Higgsless projects and G. Giudice, A. Pomarol and R. Rattazzi for our construction of the effective description of composite Higgs models. A lot of material presented during this talk is based on two on-going projects with R. Contino, M. Moretti, F. Piccinini and R. Rattazzi and with J.R. Espinosa and M. Mühlleitner and these words are to tell them all my gratitude for their support. I also thank the organizers of the EPS-HEPP'09 conference and the board of the EPSHEPP division for inviting me to present this review talk. My work has been supported in part by the 'MassTeV' ERC advanced grant 226371.

\section{References}

[1] R. Barate et al. [LEP Working Group for Higgs boson searches], Phys. Lett. B 565 (2003) 61 [arXiv:hep-ex/0306033].

[2] C. Amsler et al. [Particle Data Group], Phys. Lett. B 667 (2008) 1.

[3] A. Hoecker, these conference proceedings, arXiv:0909.0961 [hep-ph].

[4] S. Heinemeyer, W. Hollik, D. Stockinger, A. M. Weber and G. Weiglein, JHEP 0608 (2006) 052 [arXiv:hep-ph/0604147].

[5] G. F. Giudice and R. Rattazzi, Nucl. Phys. B 757 (2006) 19 [arXiv:hep-ph/0606105].

[6] J. A. Casas, J. R. Espinosa and I. Hidalgo, JHEP 0401 (2004) 008 [arXiv:hep-ph/0310137]. 
[7] P. Fayet, Nucl. Phys. B 113 (1976) 135.

[8] P. Batra, A. Delgado, D. E. Kaplan and T. M. P. Tait, JHEP 0402 (2004) 043 [arXiv:hep-ph/0309149].

[9] A. Birkedal, Z. Chacko and M. K. Gaillard, JHEP 0410 (2004) 036 [arXiv:hep-ph/0404197].

[10] A. Brignole, J. A. Casas, J. R. Espinosa and I. Navarro, Nucl. Phys. B 666 (2003) 105 [arXiv:hep-ph/0301121].

[11] M. Dine, N. Seiberg and S. Thomas, Phys. Rev. D 76 (2007) 095004 [arXiv:0707.0005 [hep-ph]].

[12] A. Strumia, Phys. Lett. B 466 (1999) 107 [arXiv:hep-ph/9906266].

[13] K. Blum, C. Delaunay and Y. Hochberg, arXiv:0905.1701 [hep-ph].

[14] K. Blum and Y. Nir, Phys. Rev. D 78 (2008) 035005 [arXiv:0805.0097 [hep-ph]].

[15] N. Bernal, K. Blum, Y. Nir and M. Losada, JHEP 0908 (2009) 053 [arXiv:0906.4696 [hep-ph]].

[16] A. Uranga, these conference proceedings.

[17] N. Arkani-Hamed, A. G. Cohen and H. Georgi, Phys. Lett. B 513 (2001) 232 [arXiv:hep-ph/0105239].

[18] N. Arkani-Hamed, A. G. Cohen, E. Katz and A. E. Nelson, JHEP 0207 (2002) 034 [arXiv:hep-ph/0206021].

[19] B. Grinstein and M. Trott, JHEP 0811 (2008) 064 [arXiv:0808.2814 [hep-ph]].

[20] H. C. Cheng and I. Low, JHEP 0309 (2003) 051 [arXiv:hep-ph/0308199].

[21] M. S. Carena, J. Hubisz, M. Perelstein and P. Verdier, Phys. Rev. D 75 (2007) 091701 [arXiv:hep-ph/0610156].

[22] T. Han, H. E. Logan, B. McElrath and L. T. Wang, Phys. Rev. D 67 (2003) 095004 [arXiv:hep-ph/0301040].

[23] M. Perelstein, M. E. Peskin and A. Pierce, Phys. Rev. D 69 (2004) 075002 [arXiv:hep-ph/0310039].

[24] M. S. Chanowitz and M. K. Gaillard, Nucl. Phys. B 261 (1985) 379.

[25] R. Contino, C. Grojean, M. Moretti, F. Piccinini and R. Rattazzi, to appear.

[26] K. Agashe, R. Contino and A. Pomarol, Nucl. Phys. B 719 (2005) 165 [arXiv:hep-ph/0412089].

[27] I. Low, R. Rattazzi and A. Vichi, arXiv:0907.5413 [hep-ph].

[28] J. M. Cornwall, D. N. Levin and G. Tiktopoulos, Phys. Rev. Lett. 30 (1973) 1268 [Erratum-ibid. 31 (1973) 572].

[29] C. T. Hill and E. H. Simmons, Phys. Rept. 381 (2003) 235 [Erratum-ibid. 390 (2004) 553] [arXiv:hep-ph/0203079].

[30] H. Georgi and D. B. Kaplan, Phys. Lett. B 145 (1984) 216.

[31] G. F. Giudice, C. Grojean, A. Pomarol and R. Rattazzi, JHEP 0706 (2007) 045 [arXiv:hep-ph/0703164].

[32] R. Barbieri, B. Bellazzini, V. S. Rychkov and A. Varagnolo, Phys. Rev. D 76 (2007) 115008 [arXiv:0706.0432 [hep-ph]].

[33] K. Agashe and R. Contino, arXiv:0906.1542 [hep-ph].

[34] A. Azatov, M. Toharia and L. Zhu, Phys. Rev. D 80 (2009) 035016 [arXiv:0906.1990 [hep-ph]]. 
[35] G. L. Bayatian et al. [CMS Collaboration], "CMS technical design report, volume II: Physics performance,” J. Phys. G 34 (2007) 995.

[36] J.R. Espinosa, C. Grojean and M. Muehlleitner, to appear.

[37] A. V. Manohar and M. B. Wise, Phys. Lett. B 636 (2006) 107 [arXiv:hep-ph/0601212].

[38] A. Pierce, J. Thaler and L. T. Wang, JHEP 0705 (2007) 070 [arXiv:hep-ph/0609049].

[39] M. Duhrssen, ATL-PHYS-2003-030.

[40] M. Duhrssen, S. Heinemeyer, H. Logan, D. Rainwater, G. Weiglein and D. Zeppenfeld, Phys. Rev. D 70 (2004) 113009 [arXiv:hep-ph/0406323].

[41] G. Aarons et al. [ILC Collaboration], arXiv:0709.1893 [hep-ph].

[42] J. A. Aguilar-Saavedra et al. [ECFA/DESY LC Physics Working Group], arXiv:hep-ph/0106315.

[43] http://clic-study.web.cern.ch/

[44] V. Barger, T. Han, P. Langacker, B. McElrath and P. Zerwas, Phys. Rev. D 67, 115001 (2003) [arXiv:hep-ph/0301097].

[45] A. Falkowski, S. Pokorski and J. P. Roberts, JHEP 0712 (2007) 063 [arXiv:0705.4653 [hep-ph]].

[46] J. Bagger et al., Phys. Rev. D 52 (1995) 3878 [arXiv:hep-ph/9504426].

[47] J. M. Butterworth, B. E. Cox and J. R. Forshaw, Phys. Rev. D 65 (2002) 096014 [arXiv:hep-ph/0201098].

[48] A. Ballestrero, G. Bevilacqua, D. B. Franzosi and E. Maina, arXiv:0909.3838 [hep-ph].

[49] C. Csaki, C. Grojean, H. Murayama, L. Pilo and J. Terning, Phys. Rev. D 69 (2004) 055006 [arXiv:hep-ph/0305237].

[50] M. Papucci, arXiv:hep-ph/0408058.

[51] R. S. Chivukula, D. A. Dicus and H. J. He, Phys. Lett. B 525 (2002) 175 [arXiv:hep-ph/0111016].

[52] C. Csaki, C. Grojean, L. Pilo and J. Terning, Phys. Rev. Lett. 92 (2004) 101802 [arXiv:hep-ph/0308038].

[53] A. S. Belyaev, R. S. Chivukula, N. D. Christensen, E. H. Simmons, H. J. He, M. Kurachi and M. Tanabashi, arXiv:0907.2662 [hep-ph].

[54] R. Casalbuoni, S. De Curtis, D. Dominici and R. Gatto, Phys. Lett. B 155 (1985) 95.

[55] R. Barbieri, A. Pomarol and R. Rattazzi, Phys. Lett. B 591 (2004) 141 [arXiv:hep-ph/0310285].

[56] G. Cacciapaglia, C. Csaki, C. Grojean and J. Terning, Phys. Rev. D 70 (2004) 075014 [arXiv:hep-ph/0401160].

[57] G. Cacciapaglia, C. Csaki, C. Grojean and J. Terning, Phys. Rev. D 71 (2005) 035015 [arXiv:hep-ph/0409126].

[58] R. Foadi, S. Gopalakrishna and C. Schmidt, Phys. Lett. B 606 (2005) 157 [arXiv:hep-ph/0409266].

[59] S. Dawson and C. B. Jackson, Phys. Rev. D 79 (2009) 013006 [arXiv:0810.5068 [hep-ph]].

[60] C. Csaki and D. Curtin, Phys. Rev. D 80 (2009) 015027 [arXiv:0904.2137 [hep-ph]].

[61] G. Cacciapaglia, C. Csaki, C. Grojean, M. Reece and J. Terning, Phys. Rev. D 72 (2005) 095018 [arXiv:hep-ph/0505001]. 
[62] G. Cacciapaglia, C. Csaki, G. Marandella and J. Terning, JHEP 0702 (2007) 036 [arXiv:hep-ph/0611358].

[63] K. Agashe, R. Contino, L. Da Rold and A. Pomarol, Phys. Lett. B 641 (2006) 62 [arXiv:hep-ph/0605341].

[64] G. Cacciapaglia, C. Csaki, G. Marandella and J. Terning, Phys. Rev. D 75 (2007) 015003 [arXiv:hep-ph/0607146].

[65] A. De Roeck et al., arXiv:0909.3240 [hep-ph].

[66] A. Birkedal, K. Matchev and M. Perelstein, Phys. Rev. Lett. 94 (2005) 191803 [arXiv:hep-ph/0412278].

[67] C. Englert, B. Jager and D. Zeppenfeld, JHEP 0903 (2009) 060 [arXiv:0812.2564 [hep-ph]].

[68] A. Martin and V. Sanz, arXiv:0907.3931 [hep-ph]. 